\title{
LÉ GISLATION
}

\section{L'aménagement des chutes d'eau et la Loi du 16 Octobre 1919}

\author{
Conférence faite à la Chambre de Commerce de Grenoble, le 14 Janvier
}

par M. L'Hunluer, Avocal à la Cour d'Appel de Grenoble

I.es problèmes que met en jeu l'aménagement d'une chute d'eau ne sont pas seulement des problèmes d'ordre lechnique, mais encore d'ordre juridique.

I.établissement des ouvrages de prise d'eau et de dérivation, de l'usine elle-même el du canal de fuite peuvent nécessiter l'occupalion, temporaire ou définilive, par l'exploitant de la chute, de parcelles de terrain appartenant à des tiers; la modification du régime du cours d'eau en aval de la prise peut porter préjudice à l'exercice du droit d'usage que l'article 644 du Code civil accorde aux proprietaires riverains sur l'eau des cours d'eau qui ne dépendent pas du domaine public de l'Etat.

Lntre ces divers propriétaires et l'industriel qui se propose d'aménager la chule, s'élève un conflit d'intérèts dont la solution est du domaine du droit.

Il y a peu d'amnées encore, cette solution relevait du droit commun; c'est-à-dire qu'elle était conditionnée par le principe de l'inviolabilité de la propriété privée. Nul ne pouvait entreprendre l'aménagemenl d'une chute sans l'assentiment des propriétaires intéressés; et je n'ai pas besoin de vous rappeler que ces propriétaires - ou les spéculateurs qui s'étaient substitués a eux - savaient faire payer fort cher leur assentiment!

I.es tribunaux avaient toutefois apporté aux industriels une aide efficace dans leur lutte contre les entreprises des "barreurs de chute \%. Depuis un arrêt célèbre rendu en 1901 par la Cour de Grenoble, la jurisprudence avait réussi, par une application large et éclairée des textes mêmes du Code civil, à rendre leur opposition pratiquement inefficace dans la plupart des cas où le conflit portait uniquement sur des droits d'eau. Mais tomles les fois que les travaux d'aménagement comportaient une atteinte matérielle à des propriétés, les indusIriels ne disposaient d'aucun moyen juridique qui leur permil de passer outre aux prétentions de leurs propriétaires; ils devaient s'incliner ou renoncer.

A côté des inconvénients inhérents à l'application du droit commun, l'ancien régime des chutes d'eau offrait cependant à l'industrie des forces hydrauliques d'incontestables avantages. L'aménagement d'une chute d'eau constituait une opération purement privée, à laquelle l'Administration n'intervenait que dans la mesure strictement nécessaire à la sauvegarde de l'hygiène et de la sécurité publiques. L'industriel restait maitre chez lui, étant - ou tout au moins pouvant légitimement se croire, car les événements sont venus lui donner tort - propriétaire de son usine, définitivement et sans restriction.

Depuis la loi du 16 octobre 1919, au contraire, la solution du problème juridique de l'aménagement des chutes d'eau a cessé d'appartenir au droit commun. Toutes les fois qu'il s'agit d'une chute de quelque importance - et ce cas est le seul dont je me propose de vous entretenir ce soir - l'intérèt général prévaut sur le droil du propriétaire; l'industriel qui se propose d'aménager une chute ne court plus le risque d'être arrêté par les prétentions exhorbitantes ou par l'opposition des «barreurs de chute». Par contre, l'entreprise qu'il exploite n'est plus une entreprise purement privée; concessionnaire de l'Etat, il est censé travailler pour le comple de l' $\Lambda$ dministration; il est soumis à un contrôle permanent et assujet $\mathrm{t}$ à des charges variées.

La législation nouvelle a donc fait subir au problème juridique de l'aménagement des chutes d'eau, une transformation complète C'est sur cette transformation, envisagée d'un point de vue essentiellement pratique, que je me propose ce soir de retenir votre attention, en mettant en parallèle les avantages et les inconvénients qu'elle présente pour l'industrie des forces hydrauliques et en recherchant les répercussions qu'elle a pu-entraîner dans l'ordre économique.

Voici plus de sept ans déjà que le nouveau statut des forces hydrauliques françaises est en vigueur. Et cependant, il est incontestable que la loi de 1919 est encore aujourd'hui mal connue et mal comprise.

Mal connue, d'abord. Et cela, parce que peu appliquée. Contrairement aux prévisions optimistes de ses auteurs, la loi de 1919 n'a reçu jusqu'ici, en effet, que peu d'applications pratiques. En particulier, le nombre des concessions accordées et mises en œuvre dans notre région depuis sa promulgation est resté extrèmement restreint. Vous me permettrez de citer ici quelques chiffres : dans notre département de I'Isère, berceau de la houille blanche, deux concessions seulement ont été accordées à l'heure actuelle, et encore, lune d'elles, celle du Chambon, présente ce caractère particulier qu'elle porte sur un barrage isolé et non sur une chute complète. Dans la Savoie, mieux partagée, on relève trois concessions; dans la Haute-Savoie, une seule. Pour l'ensemble de la région des Alpes, on ne compte actuellement que neuf concessions accordées sous le régime de la loi de 1919 .

J'aurai à revenir tout à l'heure sur les causes de cette stagnation, de cette crise - il faut bien dire le mot - de l'aménagement des forces hydrauliques. Mais c'est là, de toule évidence, une situation anormale qui ne saurait se prolonger indéfiniment : le jour n'est sans doute pas éloigné où les chantiers s'ouvriront à nouveau, et le moment est peut-ètre opportun de rappeler l'attention des intéressés sur la législation à laquelle seront soumises leurs entreprises.

Peu appliquée au point de vue administratif, la loi de 1919 l'a été moins encore, jusqu'ici, au point de vue judiciaire. Le contentieux du nouveau régime des forces hydrauliques est encore à l'état embryonnaire. Le nombre des décisions publiées à l'heure actuelle et qui s'y rapportent atteint à peine, à ma conmaissance, une demi-douzaine, parmi lesquelles figurent un seul arrèt de la Cour de Cassation et un seul arrèt du Conseil d'Etat.

Nal comnue, la loi de 1919 est aussi - j'ai eu à plusieurs reprises l'occasion de le constater - imparfaitement comprise. Et, s'il en est ainsi - et c'est là une constatation qu'il est déplorable d'avoir à faire au début d'un tel exposé - s'il en est ainsi, cela tient pour beaucoup à ce que c'est une loi mal faite. Mal faite, en dépit - - et, chose singulière, peut-ètre même à cause du soin apparent apporté à sa préparation, parce que cette préparation s'est trouvée viciée par une erreur fondamentale de méthode. 
La Commission extra parlementaire qui en avait été chargéc a eu le fort d'élaborer un projet sans prendre le soin d'en coordonner les dispositions en les appuyant sur quelques principes généraux bien definis : méthode legislative déplorable, mais cependant voulue, et, il faut bien le reconnaître, rendue peut-ètre inévitable par la procédure mème qui avait été adoptéc. Il sagisait avant tout daboutir, el daboutir rapidement. (or, il était de toute évidence que rien ne seraït plus difficile que d'obtenir l'unanimité sur des définitions de principe dans une assembléc où se rencontralent, à còté des représenlants de l'industrie hydroélectrique, des directeurs de ministères el des parlementaires de toute nuance, y compris les plus avancées. Par contre, un accord pourait à la rigueur les réunir sur des textes transactionnels.

Or, en matière législative, rien n'est plus détestable qu'une Iransaction. Qui dit transaction dit presque toujours équivoque, et trop souvent contradiction.

Cette absence d'un plan d'eusemble, d'une idée directrice suffisamment nette, rend particulièrement. ingrate la tàche de l'interprète de la loi de 1919. C'est cette idée directrice. l" "intention du législateur "-.. pour employer le langage des théoricıens du droit - qui permet d'ordinaire de suppléer anx lacunes qui sont inévitables dans toute la loi, mème la mieux étudice. Or, I'intention du législateur de 1919 est, dans son ensemble, singulièrement malaisée de discerner. Parfois on est obligé de constater son absence; parfois mème -... et ceci est plus grave on la surprend en contradiction avec elle-mème (1).

\section{$*^{*} *$}

Mais, quels que soient ses défauts, la loi de 1919 n'en conslitue pas moins, pour l'industrie des forces hydrauliques, un instrument de travail, imparfait sans doute, mais qui marque néanmoins un incontestable progrès sur l'état de choses antérieur pour tout ce qui touche à la solution du conflit d'intérêts que l'aménagement de toute chute suscite entre l'industrie et la propriéte. Comme je vous le disais tout à l'heure, depuis 1919 , les termes du problème sont renversés : l'intérêt général prime actuellement le droit du propriétaire; l'acte - - loi ou décret qui investit le concessionnaire du droit d'aménager et d'exploiter la chute concédée, lui confère une véritable délégation de la puissance publique qui lui permet de passer outre à l'opposition des particuliers ou à leurs prétentions excessives. Théoriquement, une concession de forces hydrauliques pourrait être instituée et mise en cuvre sans que son titulaire ait obtenu ou même seulement sollicité l'assentiment d'aucun des propriétaires intéressés.

Est-ce à dire pour cela qu'il n'est pas préférable, en pratique, d'obtenir cet assentiment ? Certes non. Vous savez tous aussi bien que moi qu'un mauvais arrangement vaut souvent mieux qu'un bon procès. Mais, ce qu'il importe de ne jamais perdre de vue, c'est qu'un accord amiable n'est plus désormais indispensable; qu'il ne présente donc un intérêt que dans la mesure où les conditions mises à sa conclusion sont normales et équitables.

C'est là un point sur lequel il me paraît nécessaire d'insister. J'ai eu, en effet, à plusieurs reprises, loccasion de constater la survivance d'anciens errements qui n'ont plus actuellement de raison d'ètre.

L'intérêt que peut présenter pour l'industriel qui se propose d'aménager une chute d'eau -..- concessionnaire ou simple de-

(1) C'est notamment le cas en ce qui concerme les dispositions de la loi relatives à la participation de l'Etat, en fin de concession, sur les incidents d'actif de la société concessionnaire, lorsque le cahier des charges prévoit le calcul des redevances proportionnelles en fonction des "dividendes ou bénéfices répartis. "Voir sur ce point .J. L'Huillier, Législation des Forces hydrauliques (Allin Michel, éditeur, 1926), p. 123 à 126 et p. 138. mandeur en concession --. la conclusion d'un accord amiable, les conditions moyennant lesquelles un lel accord peut être considére comme avantageux, different dailleurs notablement suivant l'objel de cel aceord.

Il esl évidemment impossible, dans un exposé tel que celui que j'ai entrepris ec soir, d'envisager l'infinie variéle des cas particuliers, de prévoir toules les questions dopportunité qui peuvent influer sur la decision à prendre. Mais il me semble néanmoins possible de tracer - sous la réserve, je le répête, des circonslances de fail particulières a chaque espece - un certain nombre de direclives génerales correspondant aux trois hypothèses principales qui sont susceplibles de se rencontrer dans la pratique.

Il se peut, lout d'abord, qu’il sagisse de traiter avec les propriétaures de lemains nécessaires à l’élablissement des ouvrages de la chute ou destinés a elre submergés par la reteme du barrage. En pareil cas, la loi prévoil trois solutions:

10 En règle générale, l'oceupation à tilre de servilude, moyennant une indemnite préalable fixée, à délaul daceord, par les tribunaux civils ;

$2^{\circ}$ L'acquisition obligatoire des termins occupes, le prix étant également fixé, s'il y a lieu, par la juridiction civile, lorsqu'il y a "privation de la jouissance du sol au dola du terme" prévu pour l'achèvement des travaux;

$3^{\circ}$ Enfin, l'expropriation, dans les cas particuliers prevus par l'article 5 et lorsque le concessionnaire a obtenu la déclaration d'utilité publique - qui, d'ailleurs, ne lui est pratiquement jamais refusée.

C'est évidemment, à mon avis, l'hypothese dans laquelle un accord amiable présente le plus d’intérèt. La valeur vénale de la servilude ou de la propriété qu il s'agit dacquérir esl d'ordinaire aisée à évaluer; il en est de mème de la majoration qu'il peut être équitable de lui faire subir pour compenser le dommage ou mène seulement la gène apportée au propriétaire par son éviction; de ce que l'on pourrail appeler, en un mot, le prix de la contrainte. Par ailleurs, un procès entraîne nécessairement des frais : l'évaluation de l'indemnité par des experts et surtout par le jury d'expropriation ne va pas sans aléas. L'accord n'est donc à déconseiller que lorsque les prétentions des propriétaires sont réellement exorbilantes.

Et, mème dans ce cas, il peut arriver que l'industriel ait intérêt à traiter à l'amiable. Il suffit de supposer que des besoins urgents d'énerşie l'aient obligé à commencer les travaux - à ses risques el périls - avant que la concession ne lui ait été accordée. Il lui faudra alors, comme sous le régime ancien, s'incliner ou renoncer; car un simple demandeur en concession ne dispose d'aucune des prérogatives altachées par la loi au titre de concessionnaire, exception faite du droit de pénétration pour études dans les propriétés privées.

Une deuxième hypothèse est celle où l'industricl est en présence de droits de riveraineté exercés, c'est-à-dire dont l'existence se manifeste par une ulilisation effective el régulière des caux de la chute : irrigations, moulins, ou même usine de quelque importance aménagé sous le régime ancien.

Dans ce cas aussi, il y a généralement intérè à traiter amiablement. Mais, pour definir les conditions dans lesquelles un accord peut être considéré comme normal et équilable, il est indispensable de bien préciser l'élendue des droils respectifs du concessionnaire et celle du riverain, telle qu'elle résulte des dispositions de l'article 6 de la loi. Ceci va m'obliger à entrer dans quelques délails qui vous paraîtront peut-être quelque peu arides, mais que je crois nécessaires. Il s'est produit, en effet, dans la pratique, de véritables abus qui ne peuvent s'expliquer que par une inter- 
prétation inexacte de la loi de la part des concessionnaires qui en ont été victimes.

L'article 6 de la loi du 16 octobre 1919 stipule que "l'éviction des droits particuliers à l'usage de l'eau... donne lieu à une indemnite en nature ou en argent.... "Lorsqu'il s'agit de droits exercés, cette indemnilé se présente, en principe, sous la forme de la restilulion de l'eau ou de l'énergie précédemment utilisées. Que laut-il entendre par là ? La question n'est pas, je dois le dire, sans dommer lieu à quelques controverses.

Avant 1919, il arrivait déjà parfois que la cession de certains droits de riveraineté élail rémunérée par un paiement en nature sous forme de restitution d'eau ou d'énergie. Mais le riverain élait alors maître d'imposer ses conditions. Il pouvait exiger de l'industriel auquel il cédait ses droits une amélioration de sa situation anlérieure, telle que l'accroissement ou la régularisation de la puissance utilisée. En est-il de même actuellement? Je ne le crois pas.

Sous l'empire de la loi de 1919 , le riverain a droit á la restiLution de l'eau ou de l'énergie à titre d'indemnité. Or, qu'est-ce qu'une indemnité ? C'est, par définition, la réparation d'un préjudice; réparation qui doit comprendre toute l'étendue du dommage correspondant, mais rien de plus. Le concessionnaire doit donc assurer au riverain une situation équivalente à celle dont il jouissat avant l'instilution de la concession; mais il ne serail en ancun cis tenu de le faire bénéficier gratuitement d'avanlages nouveaux.

C'est ainsi que, en matière de restitution d'énergie - c'est le seul cas où des intérêts vraiment importants soient ordinairement en jeu -- on doit, à mon avis, considérer comme anormales des stipulations prévoyant :

-. Soit la restitution d'une puissance supérieure à celle aménagie par le riverain ;

- Soit la restitution d'une puissance égale à celle dont il pouvait disposer en hautes eaux, mais régularisée ;

- Soit mème enfin la restitution à titre entièrement gratuit de l'énergie utilisée, lorsque la substitution de l'énergie restituée a la force motrice du cours d'eau a pour effet de procurer au riverain une économie notable dans ses frais d'exploitation (1).

Il ne faut évidemment pas en conclure que le concessionnaire ou le demandeur en concession n'aura pas intérêt, dans bien des cas, et surtout lorsqu'il s'agit d'une puissance minime, à accorder au riverain quelques avantages supplémentaires qui lui permettront d'éviter les frais et les aléas d'un procès. Mais l'intérèt d'un accord amiable disparaitt entièrement, lorsque l'acceptation des prétentions du riverain aurait pour effet d'imposer à l'entreprise une charge excessive : charge d'autant plus lourde qu'elle est susceptible de peser sur la concession pendant toute sa durée.

On a vu dans la pratique - et c'est pourquoi je crois utile d'insister sur ce point - des concessionnaires, sans doute mal informés, accepter des conventions réellement inadmissibles.

L'acceptation de conditions excessives ne se justifierait même pas ici, d'ordinaire, par la nécessitè de gagner du temps, comme cela peut avoir lieu en matière d'acquisition de servitudes ou de terrains. En effet, le droit à indemnité du riverain ne prend naissance que du jour où ce riverain éprouve un préjudice, c'est-àdire du jour où sa dérivation cesse d'être alimentée normalement. Or, ceci suppose d'ordinaire que l'usine nouvelle fonctionne,

(1) On observera, d'ailleurs, qu'une fois la concession accordéc, il n'est plus au pouvoir du riverain de s'opposer à la mise en route de l'usine, même si, au jour de cette mise en route, la nature ou le montant de l'indemnité à laquelle il a droit sont encore en litige. Il pourrait seulement, en ce cas, obtenir le fourniture immédiate de la quantité d'eau ou d'énergie nécessaire à son exploitation, à sitre de mesure provisoire et par voie d'ordonnance de référé. et il y a tout lieu de croire que, quelque hâte que mette l'industriel à achever scs travaux, il aura pu obtenir la concession avant de faire tourner ses turbines (1). Il est vrai que le contraire s'est vu parfois...

Reste une troisième hypothèse : celle ou il s'agit de droits de riveraineté non exercés. Le droit existe, en quelque sorte, à l'état abstrait, par le seul point qu'un fond coule un cours d'eau ou est traversé par ce cours d'eau, mais le propriétaire laisse couler la rivière sans chercher à l'utiliser. Ici, la situation est entièrement différente; à part certains cas particuliers, l'industriel n'a pas intérêt à traiter amiablement avec le riverain.

En effet, aux termes de l'article 6 de la loi, l'indemnité à laquelle donne lieu l'éviction des droits à l'usage de l'eau non exercés est fixée par l'acte de concession. Pratiquement, le décret qui approuve la concession détermine le taux de cette indemnité en francs par mètre de rive.

Il me semble utile de préciser les conséquences de cette fixa tion administrative de I'indemnité. Si l'on s'en réfère à une très ancienne jurisprudence relative à la matière extrêmement voisine de la redevance tréfoncière des mines, on doit admettre que les dispositions de l'article 6 ont pour effet de mettre hors du commerce les droits de riveraineté non exercés - du moins en tant qu'il s'agit de l'aménagement d'une chute concessible; ce qui entraîne la nullite de plein droit de tous les contrats relatifs à ces droits conclus postérieurement à la promulgation de la loi entre les riverains et uu concessionnaire ou un demandeur en concession. Par ailleurs, le taux de l'indemnité étant fixé souverainement par l'Administration, son montant ne peut pratiquement donner lieu à aucune contestation.

Ln accord amiable sur cette indemnité serait donc, en règle générale, tout à la fois sans valeur juridique et sans utilité pratique. Il peut cependant se faire qu'un demandeur en concession ait intérêt à s'assurer, moyennant quelques menus avantages stipulés dans un accord particulier, les bonnes grâces de certaines communes riveraines ou mème de certains riverains influents. La pratique administrative admet que de tels accords peuvent être homologués par l'acte de concession, si leurs conditions ne paraissent pas excessives à l'ingénieur en chef chargé de l'instruction de la demande. La sanction administrative qu'ils reçoivent ainsi couvre la nullité dont ils sont entachés à l'origine. Mais, je le répète, il ne s'agit là que de cas particuliers. En principe, l'abstention doit être ici la règle.

J'ai supposé jusqu'à présent que l'industriel avait à traiter avec les propriétaires proprement dits des terrains intéressés par l'aménagement de la chute. Mais il se peut également qu'il se trouve en présence d'un tiers industriel ou intermédiaire qui aurait acquis les droits de ces propriétaires soit en vue d'aménager la chute lui-même, soit dans un but de spéculation. Sa situation ne se trouve pas pour cela sensiblement modifiée; l'achat du "portefeuille " constitué par le ticrs n'est plus ni nécessaire, ni suffisant pour obtenir la concession et la mettre en œuvre; il n'a donc généralement intérèt à traiter avec ce tiers que dans la mesure et dans les conditions où il aurait eu intérêt a obtenir un accord amiable des propriétaires primitifs.

Il me paraît utile, à ce sujet, de rappeler les règles admises dans la pratique administrative en ce qui concerne la fixation des indemnités relatives aux droits de riveraineté non exercés précédemment acquis par des tiers. D'après la circulaire ministérielle du 31 juillet 1922, lorsque ces droils ont été acquis avant la promulgation de la loi, et que cette acquisition a été faite en vue d'aménager la chute et non dans un but de spécula-

(1) Sur ce cas, voir J. L'Huillier, Les restitutions en nature à la charge des concessionnaires de forces hydrauliques. Revue générale de l'Electricité, 11 décembre 1926, p. 909. 
tion, lacte de concession peut alloner aux accquéreurs évincés une indemnité égale aux déboursés qu'ils justifient avoir effectués. Dans tous les autres cas, c'est le droit commun qui s'applique; c'est-à-dire que lintermédiaire ne saurait réclamer aulre chose que lindemnite forfaitaire de $X$ franes par melre de rive à laquelle aurait eu droit le propriétaire primitif - en admettant tontefois qu'il se soit valablement substitué à ce propriétaire. Mais ceci est une autre question, dont l'examen m'entraînerait trop loin.

En resumé, la loi du 16 octobre 1919 assure aux industriels la certitude de n'avoir jamais à payer an dela de leur juste valeur les droits dont l'acquisition est nécessaire à l'aménagement de la chute concédée.

Mais cet avantage considérable ne leur est pas accordé gratuitement. La délégation de la puissance publique qui leur permet de vaincre les résistances des particuliers comporte une contrepartie. La qualité de concessiomare de l'Etat implique des restrictions aux libertés ordinaires de l'industrie, des charges de diverses natures. Quelle est, pour l'industric des forces hydrauliques, la portée pratique de ces charges et de ces restrictions, quelles en sont les répercussions économiques ? C'est ce quil me reste à examiner rapidement.

Il est un fait dont on ne peut manquer deetre frappé, et dont certains ont parfois conclu un peu hativement à la faillite de la loi de 1919 : cest que la mise en application de cette loi, loin d'avoir provoqué, comme lespéraient ses promoteurs, un nouvel essor dans le développement de l'industrie hydro-électrique française, a coïncidé, au contraire, avec un arrêt ou tout au moins un ralentissement très marqué de ce développement. Te vous ai domé, tout à l'heure, à ce sujet, quelques chiffres particulièrement éloquents en ce qui concerne la région des Alpes. Dans les autres régions, dont l'aménagement était moins poussé en 1919 , le nombre des concessions accordées est relativement plusélevé - ce qui ne veut pas dire, d'ailleurs, qu'il y ait eu beaucoup plus de réalisations. Mais il n'en est pas moins certain que l'aménagement des forces hydrauliques françaises subit, depuis sept ans, une crise profonde et générale.

Il est permis de se demander s'il existe un rapport entre cette crise et les charges nouvelles que la loi de 1919 a imposées aux exploitants de chutes d'eau; en d'autres termes, dans quelle mesure ces charges peuvent être considérées comme un facteur de ralentissement du développement de l'industrie des forces hydrauliques. Pour pouvoir donner à cette question, dont la gravité ne vous échappera pas, une réponse véritablement objective, il convient d'examiner une à une les principales charges imposées par la loi aux concessionnaires de forces hydrauliques et de bien préciser la portée réelle de chacune d'elles.

Avant d'entreprendre un tel examen, une observation préliminaire s'impose : la loi ne contient que le principe des restrictions et des charges par lesquelles la concession se distingue d'une entreprise industrielle purement privée. Elle trace simplement un cadre général dans lequel le cahier des charges de chaque concession doit fixer la situation particulière de l'entreprise à laquelle il s'applique. Or, ce qu'il convient d'apprécier, pour s'en tenir à ce point de vue strictement objectif auquel je me suis placé, ce n'est pas le principe, mais son application ; c'est donc moins la loi elle-mème que la pratique administrative. On peut dire que les charges imposées à l'industrie des forces hydrauliques par la loi de 1919 ne sont en elles-mêmes ni modérées ni excessives; elles peuvent être l'une ou l'autre, selon l'esprit dans lequel cette loi sera appliquée.

Ceci dit, on peut ramener à quatre chefs principaux les restrictions et les charges qui affectent les concessions de forces hydrauliques. Ce sont : le caractère temporaire du droit conféré au concessionnaire; les redevances auxquelles il est assujetti envers l'Elat; les resernes d'ean ou d'énergie qu'il peul che lenu de laisser a la disposilion des services publics ou des consommateurs locaux; enfin, les larifs maxima qui penvent hi être imposés pour la vente de l'énergie produite.

Je passerai rapidement sur le caraclere temporaire des conessions, car c'est là une restriction aussi peu gènante en pratique qu'elle parait grave en theorie. Vous savez, d'abord, que la duré maximum légale des concessions -..- qui est, en fail, leur dure normale -.- est de soixantempuinze ans. Or, une entreprise qui ne pourrat pas amortir son capital dans un pareil déai ne serait évidemment pas viable. l)aulre part, ce maximum luimème paraît avoir un caractère surtout théorique; une concession parvenue à son terme peut être renouvelée indéfiniment par periodes successives de trente ans, ef ce renouvellement purait devoir ble en quelque sorte automalique. En effet, l'arliek 13 de la loi subordomne la reprise des installations par l'Etal en fin de concession a linstitution d'une concession nouvelle, que le concessiomaire sortant a d'ailleurs le droit de se faire attribuer par priorilé. Iess auteurs de la loi ont voulu par la

les travaux préparatoires sont formels sur ce point - - assurer en quelque sorte une pérennité de fait aux concessions dont l'exploitation se poursuit conformément à l'intérêt général.

En ce qui concerne les redevances, la pratique suivie par l'Administration est loin d'échapper à lonte critique. Mais ces critiques, je me hàle de le dire, ne sont pas d'ordre économique. Elles visent la légalité de certaines formules, Jes difficultés pratiques d'application de certaines des clauses du cahier des charges-types imposé par l'Administration à loules les entreprises: elles ne s'adressenl pas, dans l'ensemble, aux taux habiluellement adoptés pour ces redevances. Or, du point de vue qui nous préoceupe, e'est bien la considération de taux qui est primordiale.

La loi prévoil deux formes distincles pour la redevance, qui peut ètre calculece en fonction, soit de la production annuelle de lusine en KWH, soit des "bénéfices ou dividendes repartis" Dans la pratique, la redevance proportionnelle a l'energic produite est slipulée dans tous les cas pour une première période de dix ans à compler de la mise en exploitation de l'usine, avec un taux rariant de $\&$ a 7 centièmes de centime par KWH régularisé, taux réduit de moitié pour les KWH de hautes eaux. C'est lá une charge très modérée, puisqu'elle représente une proportion de l'ordre de $1 \%$ au maximum du prix de revient de l'énergie produite. Pour une centrale de $10.000 \mathrm{KW}$, la dépense annuelle correspondante serait de l'ordre de 15.000 a $30.000 \mathrm{fr}$.

Après l'expiration de celte période initiale de dix ans, les formules adoptées par le cahier des charges-type font entrer en ligne de compte, dans tous les cas, le bénéfice net réalisé par le concessionnaire. C'est là une pratique dont la légalité est extrêmement douteuse, car la loi de 1919 ne permet d'imposer au concessionnaire la participation de l'Etat aux bénéfices que dans une hypothèse bien définie, celle où la concession est accordés à une société par actions " ayant pour objet principal l'établissement et l'exploitation de l'usine hydraulique ". C'est, surtout, une pratique fort gênante pour le concessionnaire qu'elle astreint à une comptabilité spéciale, et qui risque d'ètre pour lui une source de conflits fréquents avec l'Administration.

Si l'on s'en tient au point de vue économique, le jeu des formules adoplées par l'Administration peut évidemment donner aux redevances des valeurs très supérieures à celles qui correspondent à la période initiale. Mais il faut bien remarquer que l'application de ces formules suppose qu'il y a un bénélice, au sens du cahier des charges : c'est-à-dire que le prodnit net de l'entreprise est supéricur aux charges d'intérèt et d'amortissement des capilaux de loute nature qui y sont engages : autrement dil, que cette entreprise est réellement prospère. S'il n'en elait pas 'ainsi, la 
formule iniliale redeviendrait applicable, avec des taux réduits d'un quart.

En somme, les redevances proportionnelles, lelles que les chablit actuellement l'Administration, peuvent constituer pour Ies concessionnaires une sujétion gènante; mais la charge financière qu'elle représente ne devient réellement élevée que lorsque l'entreprise qui en est greve atteint un certain degré de prospérité. Elle ne peut donc être considérée comme excessive.

Les "réserves" prévues par l'arlicle 10 de la loi peuvent constiluer une entrave beaucoup plus sérieuse a l'aménagement d'une chule. Je ne parle pas ici des réserves en eau, qui n'ont généralement que peu d'importance, mais seulemenl des réserves en énergie.

I ce concessionnaire pent être tenu de réserver, soil aux services publics - expression dout l'acceptation est fort large - - soit aux consommateurs de la région représentés par les Conseils généraux des départements riverains de la chute, une puissance, fixéc par le cahier des charges de chaque concession, el qui peut légalement atteindro le quart de celle dont il dispose aux divers elals du cours d'eau. Il doit fournir l'energie correspondante à un larif réduil. qui peut même, dans certains cas, descendre au-dessous du prix de revient.

Ce qui est plus grave, c'est que les réserves peuvent être constiluées non seulement en vue de besoins immédiats, mais encore de besoins purement éventuels. Pendant cinq ans, la fraction do la puissance réservée qui n'est pas utilisée par les ayants droil reste pratiquement indisponible entre les mains du concessiommaire, puisqu'il est tenu de fournir l'énergie correspondante à première réquisition pendant deux ans, et moyennant six mois de préavis par la suite. Ce n'est qu'après l'expiration de ce délai de cinc ans qu'il recouvre, par paliers successifs, la libre disposilion des réserves non utilisées.

La stipulation de réserves de puissance exagérées peut donc présenter, pour le concessionnaire, des inconvénients assez graves; incertitude prolongée sur les conditions d'exploitation de la chute: immobilisation improductive de capitaux pendant plusicurs années. Ce sont là des éléments susceptibles de rendre aleatoire l'équilibre financier souvent délicat d'une entreprise à ses débuts, et mème à faire hésiter sérieusement des demandeurs en concession prudents.

Mais il faut se garder de généraliser. La charge représentée par les réscrves est essentiellement une question d'espèces, très lourde pour cerlaines entreprises, elle peut alre insignifiante pour d'autres. Tout dépend des prétentions manilestées par les collectivités locales et de la mesure dans laquelle ces prétentions auront été admises par l'Administration.

En fait, il est regrettable de constater que trop souvent les assemblées communales ou départementales ont tendance à faire passer des intérèts de clocher avant le véritable intérèl général, el à émettre des prétentions qui en font les véritables barreurs de chutes du nouveau régime. Quant à l'Administration - et je parle ici des bureaux du Ministère, et non des services régionaux (qui ont une vue infiniment plus large et plus exacte des necossitès de l'industrie - - elle tendrait pent-ètre aussi à entrer trop facilement dans les vues des collectivités locales.

Je dois signaler, d'ailleurs, que l'Administration est venue en quelque sorte renchérir sur la loi, en insérant dans les cahiers des charges une clause de priorité en faveur des services publics sur les disponibilités d'énergie du concessionnaire, clause dont l'application peut être fort gènante pour l'exploitation.

Restent enfin les tarifs maxima. On remarquera, lout d'abord, que ces tarifs ne sont souvent que des clauses de style du cahier des charges : en effet, ils sont applicables uniquement à l'énergie vendue aux bornes de l'usine et non à celle qui est distribuée par le concessionnaire. Il arrivera donc fréquemment qüils ne seront pas appliqués.

Il n'en est cependant pas loujours ainsi, el, dans bien des cas, le tarif maximum imposé au concessionnaire présentera une importance capitale pour l'équilibre financier de l'entreprise. Or, dans la pratique administrative, ces tarifs sont établis sur la base de l'évaluation du prix de revient de l'énergie produite, augmentée d'une marge bénéficiaire de 50 à $60 \%$. Ce mode de calcul est satisfaisant en soi; il est seulement regrettable que l'Administration se refuse actuellement à admettre l'adjonction aux tarifs ainsi établis d'un terme correctif destiné à tenir compte des modifications que les variations des conditions économiques peuvent faire subir aux prix de revient, comme cela a lieu pour les tarifs des concessions de distribution d'énergie, et qu'elle se borne à prévoir une révision décennale des tarifs primitifs. Il y a là une survivance de ce vieux préjugé, encore trop communément répandu, que "l'énergie hydraulique ne coûte rien "-.. et aussi une mécommaissance profonde des circonstances économiques actuelles.

D'ailleurs, en Cait de tarifs maxima, ce seraient plutôt les tarifs des concessions de distributions préexistantes qu'il y aurait lieu de rendre responsables de la crise de l'aménagement des forcés hydrauliques. En effet, les rnajorations subies par ces tarifs sont très loin d'avoir suivi celle du prix de revient de l'énergie susceptible d'être produite par des usines nouvelles. J'aurait tout à l'heure l'occasion de vous citer à ce sujet des chiffres réellement impressionnants.

Je dois enfin mentionner deux inconvénients dont on fait grief, à juste titre, au régime actuel.

C'est d'abord la lenteur des instructions des demandes de concession. La loi fixe bien à ces instructions une durée maximum d'un an. Mais c'est là une de ces prescriptions platoniques, qui n'ont et ne peuvent avoir aucune sanction efficace. En fait, le décret du 30 juillet 1920, qui avait compliqué comme à plaisir la procédure de ces instructions, rendait pratiquement impossible l'observation de la loi. II ne laissait aucun pouvoir propre aux services régionaux des forces hydrauliques. Tout devait passer par les bureaux du ministere; aussi les aris et les tran missions de dossiers se multipliaient ; et, pendant ce temps là, le denandeur en concession n'avait d'autre ressource, s'il élait pressé, que de commencer les travaux sans aucun titre, à ses risques et périls. Situation fort gênante pour lui, car, tant qu'elle se prolonge, il ne peut, comme je vous le disais tout à l'heure. se prévaloir d'aucune des prérogatives attachées par la loi au tilre de concessionnaire.

Un nouveau règlement vient d'apporter à la procédure d"instruction des demandes de concession de notables simplifications (1) Cette procédure reste néanmoins longue et complexe et il est à craindre que les délais légaux coniinuent à n'ètre pas observés.

Le deuxième de ces inconvénients est. l'exagération des droits d'enregistrement réclamés au concessionnaire. Ici, nous sommes dans un domaine où le législateur n'a rien prévu. L'Administration a inséré dans les actes de concession une claure de style : "I Ies droits de timbre et d'enregistrement de la présente convention et du cahier des charges amnexé sont supportés par le concessionnaire. "Mais quel droit était applicable ? Droit fixe de 7 fr. 20, ou droit proportionnel ? Le fisc s'est trouvé fort embarrassé. Il parait mème l'ètre encore à l'heure actuelle, car, lorsqu'on cherche à connaître sur cette question le point de vue de l'Administration, on n'obtient - j'en sais quelque chose! que les à peu près et des réticences. Néanmoins, un fait est certain; comme on élait en droit de s'y attendre, l'enregistre-

(1) Décret du 29 décembre 1926 ; Journal officiel du 7 janvier 1927, p. 310 . 
ment cherche à loncher lo phus possible 11 applique un droit proportionnel de $1,20 \%$ dans le calcul duquel il englobe tout ce qu'il peut trouver d'imposable dans le cahier des charges : évaluation des travaux, reserves, bhelles à poissons, etc.

Telles sont, résumées aussi brièvement que possible, les principales charges imposées par la loi de 1919 anx concessions de forces hydrauliques. Il reste à se demander quelles conclusions comporte le très rapide examen que nous venons d'en faire.

Je crois que lon peut dire en deux mols que ces charges sont, dans leur ensemblc, sinon légeres, du moins supportables, et qu'en tout cas, elles ne suffiraient pas, dans des circonstances normales, à entraver l'essor de l'industrie des forces hydrauliques. Elles ne peuvent donc pas èlre considérées comme une des causes déterminantes de la crise actuelle.

Je puis d'ailleurs apporter à l'appui de cette affirmalion un argument particulièrement éloquent, puisqu'il repose sur des chiffres.

D’après des renseignements qui m’ont eté obligeamment communiques par la Chambre Syndicale des Forces Hydrauliques, la puissance normale lotale concedée en France, at 1 er janvier 1926 , atteignait $376.000 \mathrm{KW}$, dont 70.000 a moins correspondant à des chutes commencées avant guerre. Sur ce Lotal, on évaluail à $167.000 \mathrm{KW}$ la puissance normale des chutes concédées dont l'aménagement n'était pas commencé ou dont les travaux étaient interrompus; ce qui représenle une proportion de concessions non mises en cuvre de $44 \%$ en puissance par rapport au total, et de plus de moitié si dans ce total, on laisse de côte les chutes commencées avant 1914. Qu'est-ce à dire, sinon que les concessionnaires ont rencontré ailleurs que dans le régime actuel des forces hydrauliques, régime auquel ils n'ont pas hesité à se soumettre les obstacles qui les empêchent daménager leurs concessions?

Ces obstacles - qui sont les véritables causes de la crise actuelle - sont d'ailleurs bien connus. Ils sont d'ordre à la fois technique et économique.

Il est d'abord à remarquer que les chutes qui restent à aménager actuellement sont pour la plupart des chutes chères, pour lesquelles le prix-or du KW installé dépasse de beaucoup les valeurs pratiquées avant la guerre. Les chutes nouvelles se trouveraient donc déjà désavantagées par rapport aux chutes anciennes, toutes choses égales d'ailleurs. Mais deux autres éléments interviennent pour accroitre ce désavantage : ce sont la dépréciation de la monnaie et la hausse du loyer de l'argent. Les chutes anciennes amortissent actuellement en francs-papier dépréciés des frais de premier établissement payés en francs-or, ce qui leur permet de conserver, pour quelque lemps encore, des prix de revient sans rapport avec la situation économique actuelle.

A valeur or du KW installé égale, le prix de revient du KWH serait donc plus élevé pour une usine nouvelle. La hausse du loyer de l'argent agit dans le mème sens; elle se complique d'ailleurs de l'extrême difficulté qu'éprouvent actuellement les entreprises à se procurer des capitaux d'emprunt - autre conséquence de l'instabilité monétaire.

Le jeu combiné de ces trois facleurs aboutil à ce résultal, qui peut, à première vue, paraître stupéfiant : c'est que le prix de revient de l'énergie produite par les usines hydrauliques nouvelles est en moyenne dix-huil fois plus élevé qu'il n'était en 1914, alors que le coefficient général d'augmentation des prix de gros n'est que de 6 environ, et que celui du prix de vente de l'énergie distribuée par les réseaux concédés alleint à peine 3 ! (1)

(1) Un calcul tres simple permet de se convaincre de ces chiffres. Supposons, en effet, qu'il s'agisse d'écruiper une chute pour laquelle
Dernière cause de crise, enlin, s’il est besoin d'en ajouler une: les immenses progrès réalisés depuis dix ans dans la production thermique de lénergie, progrès qui permetlent actuellement aux centrales thermiques de venir concurrencer sur place les usines hydrauliques.

Mais, si l'on doit chercher ailleurs que dans les charges imposées par la loi de 1919 aux concessions de forces hydrauliques les causes determinantes de la crise actudle, il resle permis de se demander si ces charges n'interviennent pas dans cetle crise comme un elément d'aggravation. Il serail, je crois, assez difficile de le contester. Quelque minimes que ces charges soient en elles-mèmes, elles n'en présentent pas moins, dans les circonstances actuelles, un caractère parfaitement inopportun, et il me: parait probable que les auteurs de la loi de 1919 les auraient failes moins lourdes sils avaient pu prévoir les condilions économiques de l'aménagement des chutes après-guerre. Vous n’ignorez pas que lo Parlement étudie actuellement un projet de loi lendant à la constitution d'un fonds special destiné à subventionner les concessions de forces hydrauliques, fonds qui serail alimenté par une taxe sur l'éclairage électrique.

Or, lorsqu'une industrie souftre au point d'avoir besoin du secours de l'Elat, n'est-il pas élémentaire que l'Etat commence par la dégrever des charges qu'il fait peser sur elle ? Ceci me paraîl une vérité d'évidence. Reste à déterminer comment un tel dégrèvement pourait avoir lieu.

On pourrait songer loul d'abord à proposer un remaniement de la loi de 1919, remaniement qui aurait l'avantage de faire disparaître les lacunes et les erreurs qu'elle contient. Mais ce serait une tàche de longue haleine, et rien ne prouve que le résultat en serait conforme au but poursuivi.

Mieux vaul done, sans doute, demander que ce dégrèvement soit réalise dans le cadre de la législation actuelle. Rien ne s'y oppose; en effet, comme je vous disais tout à l'heure, les charges imposées aux concessionnaires ne sont définies par la loi que dans leur principe. La pratique administrative pourrait donc les réduire jusqu'à les rendre purement nominales.

Il s'agirait seulement pour l'Administration d'abandonner rèsolument, dans l'établissement des cahiers des charges, cet esprit fiscal, cel esprit de défiance à l'égard de l'industrie privée, dont elle n'a pas encore réussi à se déparlir complètemenl: de s'opposer résolument à toutes les manifestations de cet esprit de clocher dont je vous parlais tout a l'heure; de ne plus voir, en un mot, dans l'industrie des forces hydrauliques, autre chose qu'une richesse nationale dont il convient avant tont d'encourager le développement, dussent les intérèts apparents de l'Etal en souffrir momentanément. Tels sonl, à mon avis, les principes essentiels dont devrait s'inspirer cette "politique de la houille blanche " qu'un de nos plus distingués parlementaires, M. le sénateur Milan, réclamail du Gouvernement dans un article recent.

C'est sur ce vou que vous me permettrez de terminer un exposé déjà trop long, quoique bien incomplel, en vous remerciant de la bienveillante attention que vous mavez accordee pour suivre un sujet qui, je me plais à le reconnâtre, était quelque peu ardu.

le prix-or du $\mathrm{K} W$. instahlé soil égale a 1,5 lois la moyenne d'avantguerre. lee coefficient de dépréciation de la momnaie étant de (i environ, celui de la hausse du loyer de l'argent de 2 , le prix de revient en francs-papier du $\mathrm{K}$ W H. sera multiplié par $1,5 \times 6 \times$ ? $=18$. 